Yong Li*, Yun-Deng Wu and Jingjing Wang

\title{
The crystal structure of 4-(3-chloro-4-fluorophenyl amino)-7-methoxyquinazolin-6-ol, $\mathrm{C}_{15} \mathrm{H}_{11} \mathrm{ClFN}_{3} \mathrm{O}_{2}$
}

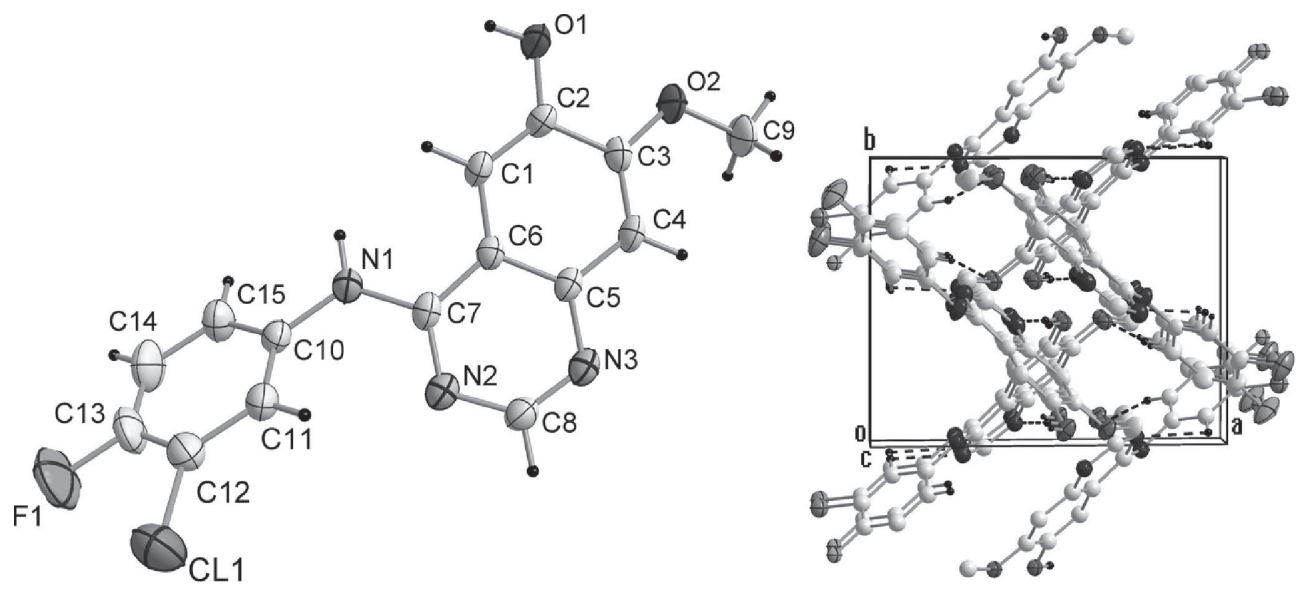

https://doi.org/10.1515/ncrs-2018-0445

Received October 21, 2018; accepted December 17, 2018; available online January 24, 2019

\section{Abstract \\ $\mathrm{C}_{15} \mathrm{H}_{11} \mathrm{ClFN}_{3} \mathrm{O}_{2}$, monoclinic, $P 2_{1} / c$ (no. 14), $a=12.9430(8) \AA$, $b=9.3196(5) \AA, \quad c=12.5959(8) \AA, \quad \beta=115.861(8)^{\circ}$ $V=1367.21(16) \AA^{3}, Z=4, R_{\mathrm{gt}}(F)=0.0512, w R_{\text {ref }}\left(F^{2}\right)=0.1359$, $T=296(2) \mathrm{K}$.}

\section{CCDC no.: 1885511}

The crystal structure is shown in the figure. Tables 1 and 2 contain details on crystal structure and measurement conditions and a list of the atoms including atomic coordinates and displacement parameters.

\section{Source of material}

The title compound is commercially available. The crystal suitable for X-ray diffraction was obtained by slow evaporation from DMF:MeOH (1:2) at room temperature.

\footnotetext{
*Corresponding author: Yong Li, Department of Chemical Engineering, Ningbo Polytechnic, Beilun District, Ningbo City 315806, Zhejiang Province, P.R. China, e-mail: 25660315@qq.com Yun-Deng Wu: Nanjing Hicin Pharmaceutical Co., Ltd, No. 1 Hengfa road, Economic and Technological Development Zone, Nanjing, P.R. China

Jingjing Wang: Department of Student Affairs, Ningbo Polytechnic, Beilun District, Ningbo City 315806, Zhejiang Province, P.R. China
}

๑ Open Access. @ 2019 Yong Li et al., published by De Gruyter. (cc) BY
Table 1: Data collection and handling.

\begin{tabular}{ll}
\hline Crystal: & Colourless block \\
Size: & $0.23 \times 0.20 \times 0.18 \mathrm{~mm}$ \\
Wavelength: & Mo $K \alpha$ radiation $(0.71073 \AA)$ \\
$\mu:$ & $0.30 \mathrm{~mm}^{-1}$ \\
Diffractometer, scan mode: & Xcalibur, $\omega$ \\
$\theta_{\max }$, completeness: & $25.4^{\circ},>99 \%$ \\
$N(h k l)_{\text {measured }}, N(h k l)_{\text {unique }}, R_{\text {int }}:$ & $5456,2497,0.027$ \\
Criterion for $I_{\text {obs }}, N(h k l)_{\text {gt }}:$ & $I_{\text {obs }}>2 \sigma\left(I_{\text {obs }}\right), 1838$ \\
$N(\text { param })_{\text {refined }}:$ & 201 \\
Programs: & Olex2 [1], SHELX [2, 3], CrysAlis \\
& {$[4]$} \\
\hline
\end{tabular}

\section{Experimental details}

The structure was solved by direct methods and refined with the SHELX crystallographic software package [2, 3]. The hydrogen atoms were placed at calculated positions with isotropic displacement parameters. The $U_{\text {iso }}$ values were fixed at 1.2 times of the parent atoms for all $\mathrm{C}(\mathrm{H})$ groups and all $\mathrm{N}(\mathrm{H})$ groups, and 1.5 times for all $\mathrm{C}(\mathrm{H}, \mathrm{H}, \mathrm{H})$ groups, all $\mathrm{O}(\mathrm{H})$ groups.

\section{Comment}

The title compound has been paid much attention because it is an analog of anti-cancer pharmaceutical Gefitinib. The crystal structures of some other derivatives of the title compound have been reported $[5,6]$.

There is one molecule in the asymmetric unit of the title structure ( $c f$. left part of the figure).

The angle between the 6-membered aromatic plane and 10 -membered aromatic heterocyclic plane is $47.38(12)^{\circ}$. This 
Table 2: Fractional atomic coordinates and isotropic or equivalent isotropic displacement parameters $\left(\AA^{2}\right)$.

\begin{tabular}{lrrrr}
\hline Atom & $\boldsymbol{x}$ & $\boldsymbol{y}$ & $\boldsymbol{Z}$ & $\boldsymbol{U}_{\text {iso }} / \boldsymbol{U}_{\text {eq }}$ \\
\hline Cl1 & $-0.14134(8)$ & $0.71513(12)$ & $0.27001(8)$ & $0.0732(3)$ \\
F1 & $-0.10006(19)$ & $0.8728(2)$ & $0.4855(2)$ & $0.0848(7)$ \\
O1 & $0.53829(16)$ & $0.0694(2)$ & $0.72281(15)$ & $0.0408(5)$ \\
H1 & 0.496095 & 0.075128 & 0.755842 & $0.061^{*}$ \\
O2 & $0.65394(15)$ & $0.0681(2)$ & $0.59872(15)$ & $0.0393(5)$ \\
N1 & $0.24197(18)$ & $0.4831(2)$ & $0.54733(18)$ & $0.0363(5)$ \\
H1A & 0.266363 & 0.434263 & 0.611614 & $0.044^{*}$ \\
N2 & $0.25849(18)$ & $0.5312(2)$ & $0.37509(18)$ & $0.0341(5)$ \\
N3 & $0.40419(18)$ & $0.4236(2)$ & $0.33419(17)$ & $0.0326(5)$ \\
C1 & $0.4136(2)$ & $0.2574(3)$ & $0.6049(2)$ & $0.0320(6)$ \\
H1B & 0.374911 & 0.260870 & 0.652099 & $0.038^{*}$ \\
C2 & $0.5028(2)$ & $0.1633(3)$ & $0.6317(2)$ & $0.0300(6)$ \\
C3 & $0.5634(2)$ & $0.1599(3)$ & $0.5606(2)$ & $0.0297(6)$ \\
C4 & $0.5297(2)$ & $0.2450(3)$ & $0.4629(2)$ & $0.0317(6)$ \\
H4 & 0.568296 & 0.240384 & 0.415601 & $0.038^{*}$ \\
C5 & $0.4365(2)$ & $0.3396(3)$ & $0.4339(2)$ & $0.0278(6)$ \\
C6 & $0.3798(2)$ & $0.3492(3)$ & $0.5061(2)$ & $0.0276(6)$ \\
C7 & $0.2917(2)$ & $0.4551(3)$ & $0.4738(2)$ & $0.0296(6)$ \\
C8 & $0.3174(2)$ & $0.5091(3)$ & $0.3114(2)$ & $0.0355(6)$ \\
H8 & 0.292991 & 0.561553 & 0.241828 & $0.043^{*}$ \\
C9 & $0.7270(2)$ & $0.0731(3)$ & $0.5407(3)$ & $0.0473(8)$ \\
H9A & 0.684979 & 0.042643 & 0.460252 & $0.071^{*}$ \\
H9B & 0.791479 & 0.010546 & 0.580295 & $0.071^{*}$ \\
H9C & 0.753846 & 0.169515 & 0.542337 & $0.071^{*}$ \\
C10 & $0.1544(2)$ & $0.5843(3)$ & $0.5284(2)$ & $0.0319(6)$ \\
C11 & $0.0613(2)$ & $0.5999(3)$ & $0.4194(2)$ & $0.0358(6)$ \\
H11 & 0.056040 & 0.544725 & 0.355754 & $0.043^{*}$ \\
C12 & $-0.0235(2)$ & $0.6971(3)$ & $0.4056(3)$ & $0.0438(7)$ \\
C13 & $-0.0164(3)$ & $0.7770(3)$ & $0.5003(3)$ & $0.0510(8)$ \\
C14 & $0.0740(3)$ & $0.7611(3)$ & $0.6092(3)$ & $0.0545(8)$ \\
H14 & 0.077737 & 0.815198 & 0.672837 & $0.065^{*}$ \\
C15 & $0.1599(2)$ & $0.6636(3)$ & $0.6236(2)$ & $0.0434(7)$ \\
H15 & 0.221345 & 0.651379 & 0.697383 & $0.052^{*}$ \\
\hline & & & &
\end{tabular}

angle is similar to the structure of the related derivative (CCDC 873811) [5], but differs from the derivative (CCDC 259805) [6], in which the two rings are almost coplanar.

In the crystal, intermolecular $01-\mathrm{H} 1 \cdots \mathrm{N} 3^{\prime}\left[{ }^{\prime}=\mathrm{x}, 1 / 2-\mathrm{y}\right.$, $1 / 2+\mathrm{z}$ ] hydrogen bonds link the molecules into a chain along the $c$ axis. Intermolecular $\mathrm{C} 15-\mathrm{H} 15 \cdots \mathrm{O}^{\prime \prime}{ }^{\prime \prime}=1-\mathrm{x}, 1 / 2+\mathrm{y}$, $3 / 2-z]$ non-classical hydrogen bonds link the molecules into a chain along the $b$ axis. These two hydrogen bonding interactions construct a network parallel to the $b c$ plane ( $c f$. right part of the figure). Intramolecular hydrogen bonds $\mathrm{C}-\mathrm{H} \cdots \mathrm{N}$ also exist in the crystal.

Acknowledgements: We gratefully acknowledge support by Ningbo Polytechnic, P.R. China for financial support.

\section{References}

1. Dolomanov, O. V.; Bourhis, L. J.; Gildea, R. J.; Howard, J. A. K.; Puschmann, H.: OLEX2: a complete structure solution, refinement and analysis program. J. Appl. Crystallogr. 42 (2009) 339-341.

2. Sheldrick, G. M.: Crystal structure refinement with SHELXL. Acta Crystallogr. C71 (2015) 3-8.

3. Sheldrick, G. M.: SHELXT - Integrated space-group and crystal-structure determination. Acta Crystallogr. A71 (2015) 3-8.

4. Agilent Technologies. CrysAlisPRO Software system, version 1.171.35.15. Agilent Technologies UK Ltd, Oxford, UK (2011).

5. Lü, S.; Zheng, W.; Ji, L.; Luo, Q.; Hao, X.; Li, X.; Wang, F.: Synthesis, characterization, screening and docking analysis of 4-anilinoquinazoline derivatives as tyrosine kinase inhibitors. Eur. J. Med. Chem. 61 (2013) 84-94.

6. Tanaka, R.; Haramura, M.; Tanaka, A.; Hirayama, N.: Structure of Gefitinib. Anal. Sci. 20 (2004) x173-x174. 